\title{
REVIEW
}

\section{Treatment of Adult Soft Tissue Sarcomas: An Overview}

\author{
Samia Arifi $\cdot$ Rhizlan Belbaraka $\cdot$ Rabie Rahhali $\cdot$ Nabil Ismaili
}

To view enhanced content go to www.rarecancers-open.com Received: June 8, 2015 / Published online: September 7, 2015

(C) The Author(s) 2015. This article is published with open access at Springerlink.com

\section{ABSTRACT}

Sarcomas are uncommon malignancies accounting for about $1 \%$ of all adult malignancies. Sarcomas are a heterogeneous group of tumors which includes more than 100 different subtypes. Surgery is the mainstay therapy for localized disease. In selected patients the combination of surgery with radiotherapy achieves better local control and offers the best chance of cure. Systemic treatment including cytotoxic chemotherapy or targeted therapies remains the mainstay therapy for most patients with advanced disease. There are a wide variety of clinical situations, such that an individualized treatment plan must be defined by a multidisciplinary tumor board. Treatment

\section{S. Arifi $(\bowtie)$}

Department of Medical Oncology, Hassan II University Hospital, Medical School, Sidi Mohamed Ben Abdellah University, Fez, Morocco

e-mail: drsarifi@yahoo.fr; samia.arifi1@smaba.ac.ma

R. Belbaraka $\cdot$ N. Ismaili

Department of Medical Oncology, Mohammed VI

University Hospital, Marrakech, Morocco

R. Rahhali

Department of Medical Oncology, Regional Center

of Oncology, Tangier, Morocco decisions should take into consideration the histology, site of disease, stage, performance status, treatment goals, and the patient's wishes. The management of patients should be carried out in a center with expertise in the treatment of sarcomas for optimal outcome. This review will cover the different treatment modalities of adult soft tissue sarcomas.

Keywords: Chemotherapy; Radiotherapy; Soft tissue sarcoma; Surgery; Targeted therapy

\section{INTRODUCTION}

Soft tissue sarcomas (STSs) are mesenchymal derived cancers which have more than 100 histological subtypes according to the most recent World Health Organization classification [1]. These tumors are rare and account for less than $1 \%$ of all adult malignancies [2]. In the USA 11,930 new cases of STS are diagnosed each year with 4870 deaths [3]. They arise from any part of the body, but the majority occur in the extremities (59.5\%) followed by the trunk (17.9\%) [4]. The most common histologic subtypes in adults are 
undifferentiated unclassified sarcoma, liposarcoma, leiomyosarcoma, synovial sarcoma, and malignant peripheral nerve sheath tumor (MPNST) [1]. Painless mass is the most common clinical presentation. Tumor tends to grow locally along tissue planes, surrounded by a so-called pseudocapsule which contains malignant cells infiltrating adjacent tissues [1]; therefore, the dissection along the pseudocapsule is contraindicated [5]. The presence of distant metastases at the time of initial diagnosis is rare [6]. The most common pattern of spread is via blood, typically to the lung [6]. Lymph node metastases are infrequent (less than 3\%) [7], with the exception of certain histologies such as epithelioid sarcoma, rhabdomyosarcoma, angiosarcoma, and clear cell sarcoma [7]. Pretreatment evaluation includes magnetic resonance imaging of the primary site and chest computed tomography [5]. Tumor stage is the most important prognostic factor. The most recent, 7th edition of the American Joint Committee on Cancer (AJCC) system is the most widely used. It incorporates tumor size, depth, lymph node involvement, distant metastases, and histologic grade in determining four stage groups with different outcome [8]. Thus, reported 5-year overall survival (OS) rates for stages I, II, and III were $90 \%, 81 \%$, and $56 \%$, respectively [8]. In addition to tumor stage, other prognostic factors are anatomic site, histologic subtype, age, and surgical margins [9]. The management of patients with STS requires a multimodality treatment provided by an expert multidisciplinary team working in a reference center or within a reference network [5]. Thereby, clinical practice guidelines recommend referral of all patients with suspected sarcoma to a reference center for appropriate diagnostic and optimal outcome [5]. In fact, Gustafson et al. [10] demonstrated that patients treated at a tumor center have better outcome as compared to patients who were not referred to a tumor center or those who were referred to a tumor center after surgery. In their series local recurrence was 2.4 times higher when patients were treated outside of a reference center and 1.3 times higher if the patients were referred to a tumor center after surgery [10].

The present review covers different treatment modalities for adult STSs based on recent clinical practice guidelines, data from clinical trials, and meta-analysis. We have excluded from this review extraskeletal Ewing sarcoma, embryonal rhabdomyosarcoma, alveolar rhabdomyosarcoma, and gastrointestinal stromal tumors (GIST), as they belong to separate therapeutic approaches. This article is based on previously conducted studies and does not involve any new studies of human or animal subjects performed by any of the authors.

\section{TREATMENT OF LOCALIZED DISEASES}

\section{Surgery}

Surgery is the standard treatment of localized STS [5] and consists of a wide surgical resection, with total en bloc excision of the primary tumor, the biopsy site, and a rim of normal tissues around the tumor [11]. Dissection along the pseudocapsule is strictly prohibited [5]. Resection margins represent the main risk factor for local recurrence [11]. The Union for International Cancer Control (UICC) recommends to report the quality of surgery in STS according to the resection type (R) with R0 as in sano, R1 as microscopic residual disease, and R2 as macroscopic residual disease. Resection type should be assessed collegially 
by pathologists and surgeons for an accurate estimation of resection margins, since the decision for re-excision or complementary treatment depends on the determination of the quality of surgery $[12,13]$. There is no consensus regarding the relevant cutoff of the minimal margin [5]. In general, $1-\mathrm{cm}$ margins are recommended; however, close margins may be necessary in some cases to preserve uninvolved major neurovascular structures [13]. Moreover, narrow margins of resistant anatomical barriers, such as muscular fasciae, periosteum, and epineurium, are likely to be adequate [5].

As regards sarcomas of the extremities, it has been demonstrated that limb-sparing surgery alone or in combination with radiotherapy (RT) in selected patients offers comparable rates of disease control and survival as amputation, as long as wide resection margins are achieved [14-16]. Therefore, primary limb amputation must be avoided in most patients. However, amputation may be the only potentially curative option in some cases including large and extensive tumors which compromise the achievement of a conservative approach with a good functional limb outcome or in case of major complications [17]. Those situations should be carefully assessed by a multidisciplinary team before carrying out amputation.

Because lymph node involvement is uncommon in STS, systematic regional node dissection is not recommended [5]. Node dissection should be performed only if there is evidence of lymph node disease [7]. In this setting, it has been reported that radical lymphadenectomy for isolated regional lymph node metastases provides long-term survival: $46 \% 5$-year survival, with a median survival of 16.3 months versus 4.3 months in patients not treated with lymph node dissection [7].
However, it is unclear if treatment of occult node metastases based on earlier detection of metastatic nodes by sentinel lymph node biopsy or positron emission tomography would improve outcome in histologies with higher frequency of lymph nodes metastasis including epithelioid sarcoma, rhabdomyosarcoma, angiosarcoma, synovial sarcoma, and clear cell sarcoma [18, 19].

Surgery alone with negative margins provides a local control rate close to $93 \%$ in a selected subset of patients (superficial and low grade tumors that are $5 \mathrm{~cm}$ or less in size, and selected truly intracompartmental tumor) [20, 21]. However, some patients are at high risk of recurrence and will require complementary treatment [22].

\section{Radiotherapy}

The benefit of RT as adjuvant treatment to limb-sparing surgery has been initially addressed in comparison with radical surgery. These studies have shown that RT, when combined with conservative surgery, provides similar rates of local control to those achieved with amputation [14]. Since the publication of these results, amputation as a primary therapy has largely been abandoned for most patients [15]. So, with the emergence of RT in the management of STSs, two randomized trials using different modalities of radiation therapy (external beam RT (EBRT) [23] or brachytherapy [24]) have been conducted in order to assess the impact of adjuvant RT on local and systemic recurrence in patients with localized STS (Table 1). The two studies demonstrated that adding RT to limb-sparing surgery reduces the risk of local recurrence by 20-25\% when compared to limb-sparing surgery alone without any advantage in OS [23, 24]. The benefit of RT was seen in high-grade and 
Table 1 Randomized trials of radiotherapy in localized adult soft tissue sarcomas

\begin{tabular}{llllll}
\hline Study & $\boldsymbol{N}$ & Treatment & Local recurrence & DFS (5 years) & OS (5 years) \\
\hline Rosenberg et al. [14] & 43 & LSS + postoperative RT & $14.8 \%$ & $71 \%$ & $83 \%$ \\
& & Amputation & $0 \%$ & $78 \%$ & $88 \%$ \\
& & & $P=0.06$ & $P=0.75$ & $P=0.99$ \\
Yang et al. [23] & \multirow{2}{*}{141} & LSS + postoperative RT & $0.01 \%$ & $\mathrm{NR}$ & $75 \%^{\mathrm{b}}$ \\
& & LSS & $23.9 \%$ & $\mathrm{NR}$ & $74 \%^{\mathrm{b}}$ \\
& & & $P=0.0001$ & & $P=0.71$ \\
Pisters et al. [24] & \multirow{2}{*}{119} & Surgery + brachytherapy & $16.7 \%$ & $83 \%^{\mathrm{a}}$ & $84 \%^{\mathrm{c}}$ \\
& & Surgery & $29 \%$ & $76 \%^{\mathrm{a}}$ & $81 \%^{\mathrm{c}}$ \\
& & & $P=0.04$ & $P=0.60$ & $P=0.65$ \\
O'Sullivan et al. [31] & \multirow{2}{*}{190} & Preoperative RT (50 Gy) & $7 \%$ & $58 \%$ & $73 \%$ \\
& & Postoperative RT (66 Gy) & $8 \%$ & $59 \%$ & $67 \%$ \\
& & $P=0.48$ & $P=0.48$ & $P=0.48$ \\
\hline
\end{tabular}

$D F S$ disease-free survival, $L S S$ limb-sparing surgery, $N R$ not reported, $O S$ overall survival, $R T$ radiotherapy

a 5 -year distant metastasis-free survival

b 10 -year overall survival in high-grade STS

c 5 -year disease-specific survival

low-grade tumors in one trial [23], whereas it was limited to high-grade tumors in the other one [24]. The identification of patients who require adjuvant RT is mandatory. Several predictor factors of local recurrence have been identified. The most important factor is surgical margins [25]. Patients with positive margins are at increased risk of local recurrence. A relative risk (RR) of 2.9 [95\% confidence interval (CI) 1.8-4.6] has been reported in patients with positive margins who did not receive adjuvant treatment [26]. Positive surgical margins have been associated with local recurrence risk even in patients treated with combined surgery and RT [9]. Re-excision seems to be the best option for favorable outcome in patients with marginal resection. Zagars et al. [27] reported local control rates of $85 \%, 85 \%$, and $82 \%$ at 5,10 , and 15 years, respectively, for patients who underwent re-resection versus $78 \%, 73 \%$, and $73 \%$, respectively, for patients who did not undergo re-resection [27]. Therefore, re-excision must be strongly considered in case of R2 or R1 resections, if adequate margins can be achieved without major morbidity $[5,13]$.

Postoperative RT improves local control in patients with marginal excisions and in those with residual tumor cells after re-excision. The 10-year local recurrence rates for patients treated with surgery alone and patients treated with combined surgery and RT was 17\% (95\% CI $\quad 8-32 \%)$ versus $53 \%$ (95\% CI $25-75 \%)$, respectively $(P=0.005)$, in patients with marginal resection and $84 \%$ in the RT group versus $37 \%$ in the no-RT group $(P=0.001)$ in patients with residual cells after re-excision [28].

Several independent adverse prognostic factors for local recurrence have been reported 
in retrospective series and population-based registries $[8,9,25,29]$. The most relevant are recurrent disease at presentation, histology, age, tumor grade, deep location, and tumor size.

A prospective nomogram which aims to estimate the risk of local recurrence for an individual patient has been established recently. The nomogram was developed from a prospective sarcoma database including 684 patients with primary, non-metastatic, extremity STS treated with limb-sparing surgery alone without adjuvant therapy. It includes five independent predictor factors of relapse: age, size, surgical margin, grade, and histology. The nomogram is useful to quantify individual 3- and 5-year risk of local recurrence; however, there is insufficient evidence to support routine use of this nomogram for clinical decision-making [22].

The recent clinical practice guidelines recommend radiation therapy as the standard treatment of lesions that are high grade, deep, and larger than $5 \mathrm{~cm}[5,13]$. There is no consensus regarding the indication of adjuvant RT for selected cases, namely STSs that are low or high grade, superficial, and larger than $5 \mathrm{~cm}$; low grade, deep, and smaller than $5 \mathrm{~cm}$; or low grade, deep, and larger than $5 \mathrm{~cm}$. So the decision should be discussed in a multidisciplinary setting and must be shared with the patient [5]. Most high-grade lesions that are deep and smaller than $5 \mathrm{~cm}$ should be treated with surgery followed by radiation therapy with exceptions to be discussed in a multidisciplinary board $[5,13]$.

RT can be administered preoperatively or postoperatively [5]. A phase III trial comparing these two modalities reported similar efficacy in terms of local control and survival [30]. After a median follow-up of 6.9 years, over $90 \%$ of patients are controlled locally, with similar rates of progression-free survival (PFS) and OS [31].
However, the two approaches differ substantially in their side effects. Acute wound complications were significantly more common with preoperative RT (35\%) as compared to postoperative RT (17\%; 95\% CI 5-30\%; $P=0.01)$. Wound healing was affected by the extent of the surgery and anatomical site of the tumor [30]. Postoperative RT induces higher rates of late complications including edema, fibrosis, and joint stiffness, which are often irreversible and adversely affect functional outcome [30]. The differences in the morbidity and functional outcome between these two approaches could be related not only to the timing of RT but also to the larger radiation field and the higher doses (66 versus 50 Gy) associated with postoperative RT [30].

The optimal timing of radiation therapy has yet to be defined. However, because of the lower rates of long-term complications and the better functional outcome reported in O'Sullivan et al.'s [30] trial there is a current trend toward preoperative RT, especially when the dose and field size are important issues. Nevertheless, postoperative RT might be preferable if severe wound-healing complications are anticipated.

Modern RT techniques such as image-guided RT and intensity-modulated RT treatment might reduce the risk of acute wound-healing problems when preoperative RT is administered and the risk of long-term side effects when postoperative RT is given [32].

The treatment should be individualized and the best option should be discussed in a multidisciplinary tumor board. A total dose of $50 \mathrm{~Gy}$ in 1.8- to 2-Gy fractions is recommended, possibly with a boost up to $66 \mathrm{~Gy}$, depending on presentation and resection margins [5].

Brachytherapy is another modality of RT in which a radiation source is placed inside the targeted area. It delivers high dose of radiation to the tumor while minimizing the dose to 
surrounding normal tissues [21]. Postoperative brachytherapy (45 Gy) reduces recurrence in high-grade STSs by 23\% [21]. There is no randomized trial comparing brachytherapy to EBRT. Further studies are needed to identify patients for whom brachytherapy may be preferred.

\section{Chemotherapy}

In spite of effective local treatment, $25 \%$ of patients will develop distant metastases $[9,33]$. Thus an effective systemic treatment to eradicate micro-metastases is strongly warranted. Over 20 randomized trials and two meta-analyses have investigated the role of adjuvant chemotherapy in localized adult STS (Table 2). Study results were conflicting. Thus, the role of adjuvant chemotherapy in patients undergoing local therapy remains unclear. The first meta-analysis by the Sarcoma Meta-analysis Collaboration published in 1997 found an improvement in local and distant recurrence-free intervals in the chemotherapy group, but no benefit in terms of OS [34]. However in the subset of patients with sarcomas of the extremities there was a statistically significant benefit in terms of OS in favor of adjuvant chemotherapy [hazard ratio (HR) for death $=0.80 ; \quad P=0.029] \quad$ [34]. This meta-analysis includes early randomized trials which used suboptimal adjuvant regimens. An updated meta-analysis including four additional new trials which used optimal dosages of doxorubicin in addition to ifosfamide confirms the limited benefit of adjuvant chemotherapy in terms of local recurrence, distant recurrence, and overall recurrence [35]. However, in contrast to the earlier meta-analysis, adjuvant chemotherapy yielded improvement in OS with an HR of 0.77 (95\% CI $0.64-0.93 ; P=0.01$ ). The benefit is further improved with regimens combining ifosfamide with doxorubicin with an absolute risk reduction of $11 \%$ (95\% CI 3-19\%; $P=0.01$ ) or a $30 \%$ versus $41 \%$ risk of death [35]. However, a survival benefit could not be found in the most recent and largest study of the European Organization for Research and Treatment of Cancer (EORTC) which was not included in the updated meta-analysis [36]. The trial randomly assigned 351 patients to receive either adjuvant chemotherapy (doxorubicin at $75 \mathrm{mg} / \mathrm{m}^{2}$ with ifosfamide at $5 \mathrm{~g} / \mathrm{m}^{2}$ ) or no additional systemic chemotherapy following surgery. The lack of efficacy might be influenced by the inclusion of patients with non-extremity sarcomas (33\%), low- and intermediate-grade tumors (55\%), and of tumors smaller than $10 \mathrm{~cm}(63 \%)$, as well as by the low dose of ifosfamide. Therefore, no definitive conclusions can be drawn. A pooled analysis combining individual patient data from this trial with another large randomized adjuvant trial found no survival benefit of adjuvant chemotherapy except in patients with marginal resection (Table 2) [37].

It is unknown whether adjuvant chemotherapy may be particularly beneficial in specific chemosensitive histologies such as myxoid, round cell liposarcoma [38] and synovial sarcoma [39]. Available data from retrospective series suggest a potential benefit of adjuvant chemotherapy in selected histologies [40, 41]; however, neither randomized clinical trials nor meta-analyses have confirmed this finding [34, 35, 37].

On the basis of these data, adjuvant chemotherapy is not standard treatment in adult-type STS. It can be proposed as an option in high-risk individual patients (with a high-grade, deep tumor larger than $5 \mathrm{~cm}$ ), but should not be considered in histological subtypes known to be chemoresistant [5]. 


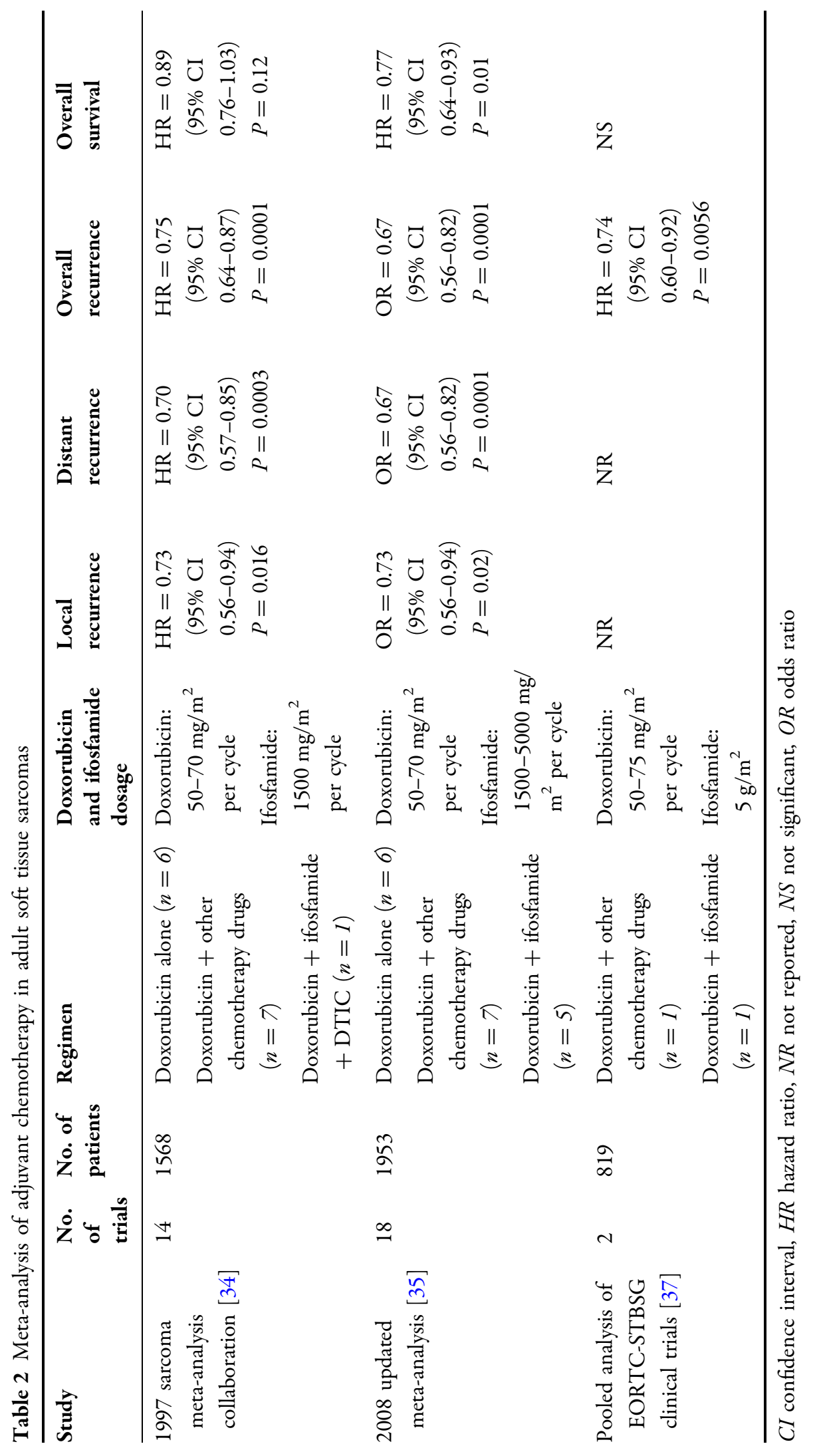


A new therapeutic approach consisting of regional hyperthermia in addition to systemic chemotherapy has been investigated in a large randomized phase III trial [42]. Patients with localized high-risk STS (G2-3, deep, at least $5 \mathrm{~cm}$ ) were randomly assigned to receive either neoadjuvant chemotherapy alone or combined with regional hyperthermia in addition to local therapy. This approach was associated with a local PFS advantage $(\mathrm{HR}=0.58 ; 95 \%$ CI 0.41-0.83) and disease-free survival benefit (HR =0.70; 95\% CI 0.54-0.92]). Response rate was higher in the group with regional hyperthermia $28.8 \%$ versus $12.7 \%$ in the group that received chemotherapy alone $(P=0.002)$. OS was better in the combined therapy group with an $\mathrm{HR}=0.66(95 \% \mathrm{CI}$ 0.45-0.98; $\quad P=0.038$ ) [43]. Thus, this therapeutic strategy offers a new therapeutic option for patients with high-risk STS including abdominal or retroperitoneal location $[5,42$, 43].

\section{PRIMARY LOCALLY ADVANCED SOFT TISSUE SARCOMAS}

For patients with unresectable primary locally advanced tumors, combined therapeutic modalities should be considered. The main objective of combined therapeutic modalities is to ovoid mutilating surgery, to improve local control, OS, and minimize sequelae.

Isolated limb perfusion (ILP) is the most investigated approach in this setting. This technique provides high concentration of antineoplastic agents locally without exposing the patient to high systemic levels of the drug by isolating the vasculature of a limb temporarily. ILP uses high doses of tumor necrosis factor alpha (TNF $\alpha$ ) and melphalan with or without interferon and is usually associated with local hyperthermia. Several groups have reported their experience with upfront ILP. Results were consistent among all these studies. The overall response rate was greater than $70 \%$ and limb salvage rate about 80\% [44-48].

Regional hyperthermia in addition to systemic chemotherapy might be a good alternative. A phase II trial which was conducted in locally advanced primary or recurrent STS found an overall response rate of $17 \%$ with a high rate of histological response and a better outcome in patients responding to the combined approach [49]. Furthermore, a subgroup analysis of the most recent phase III trial found better local PFS in patients with very large tumors (larger than $12 \mathrm{~cm}$ ) in the chemotherapy plus regional hyperthermia group as compared with patients receiving chemotherapy alone [43].

Note that both regional hyperthermia and ILP are not widely available because of the highly technical procedures required for these approaches.

Preoperative chemoradiotherapy represents another option to manage locally advanced STS [5] and available data suggest that it can be administered safely [50]. Concomitant chemoradiotherapy with low dose doxorubicin provided $67 \%$ objective response (11\% complete and $56 \%$ partial response) in 115 patients. Thirty-nine responders underwent surgery including 24 primary tumors and 15 relapses. The median survival was 29 and 50 months in responder patients [51]. Limited data exist regarding the concomitant use of ifosfamide with RT. A retrospective series of 43 patients has reported promising results that need to be confirmed by further studies [52]. Concurrent multi-agent chemotherapy (doxorubicin, ifosfamide, and dacarbazine) with RT was assessed in the Radiation Therapy 
Oncology Group (RTOG) study involving 66 patients. The 5-year rates of distant and locoregional failure (including amputation) were $28 \%$ and $22 \%$, respectively. Five-year OS was $71 \%$. But serious treatment-related toxicities were experienced in $83 \%$ of cases; $11 \%$ had major postoperative complications, and three patients experienced fatal grade 5 toxicities [53].

The benefit of neoadjuvant chemotherapy alone in the management of locally advanced STS is uncertain. In fact, it is unknown if upfront systemic chemotherapy may convert an initially unresectable tumor to a resectable one or if it may improve the rate of margin-negative resection. The single phase II trial assessing the impact of preoperative systemic chemotherapy in high-risk STS (tumors of at least $8 \mathrm{~cm}$, high grade, locally recurrent, inadequate surgery) failed to show any benefit [54]. This option could be considered in chemosensitive STS using multi-agent chemotherapy with anthracycline, ifosfamide with or without dacarbazine given to the higher response rate achieved by these protocols $[55,56]$.

In the absence of randomized controlled trials to define the most effective strategy to manage locally advanced STS, there is no consensus among reference centers and therapeutic options are usually influenced by the availability of technical equipment and the institutional experience.

\section{LOCALLY RECURRENT SOFT TISSUE SARCOMAS}

About $15 \%$ of patients with STS will develop a local relapse in spite of effective local therapy for the primary lesion $[57,58]$. Local recurrence occurs mostly within the first 2 years [57]. The outcome is poorer as compared to primary cases because of the increased risk of distant failure [59]. Wide surgical resection is the cornerstone of treatment [5]. Radiation therapy improves local control and should be considered $[5,13]$. However, achieving adequate surgical margins, salvage of the limb, and re-irradiation are often an issue in patients with recurrent STS.

Functional conservative management is always preferable but not always possible. In some cases, amputation remains the only potentially curative option, especially in previously irradiated patients.

In patients who had prior RT for their primary tumor, Indelicato et al. [60]. reported high morbidity with re-irradiation with $50 \%$ of serious complications requiring either reoperation or leading to permanent functional impairment. Brachytherapy can be an alternative for patients who had prior RT, since it provides superior rates of local control with acceptable complications [61].

Other options including neoadjuvant systemic chemotherapy or regional chemotherapy can be discussed. In fact, promising results have been reported with ILP in the management of recurrent disease. A report of 26 patients with recurrent STS, in the irradiated field, treated with TNF $\alpha$-based ILP has shown a response rate of $70 \%$. Amputation was avoided in 17 patients (65\%). Local recurrence rate was $45 \%$ in patients with multiple tumors and $27 \%$ in patients with a single tumor [62]. Regional hyperthermia combined with systemic chemotherapy might also be a good option in patients with recurrent STS. A subgroup analysis demonstrated that this new intervention results in significantly better local PFS compared with systemic neoadjuvant chemotherapy alone [43]. Further trials are needed to assess the potential benefit and the safety profile of this new 
treatment in this specific population of patients.

\section{TREATMENT OF METASTATIC SOFT TISSUE SARCOMAS}

Management of metastatic STS is a challenging problem. Treatment is essentially palliative and the potential for cure decreases drastically. The reported median OS is about 12-18 months [63, $64]$. However, about $5-8 \%$ of patients are alive progression-free 5 years after the initial diagnosis of metastasis, and most will not relapse later [65]. Chemotherapy is the mainstay of treatment in the metastatic setting. However, surgery of metastatic lesions if feasible should be offered since it provides long-term survival [66]. Reported median survival after complete excision of isolated lung metastases is $33-35$ months versus 11-13 months in patients with non-surgical treatment [66, 67]. Patients with extrapulmonary metastases can also achieve significant long-term survival when a complete resection is possible for both the pulmonary and extrapulmonary metastases [66].

Unfortunately most patients are not amenable to ablative approaches. In these instances, treatment is palliative and is based on systemic chemotherapy.

\section{Chemotherapy}

Doxorubicin alone at the dose of $75 \mathrm{mg} / \mathrm{m}^{2}$ once every 3 weeks is considered the treatment of choice in the first-line setting. It achieves a response rate of $10-25 \%$ and a median survival in the range of 1 year $[63,64]$. It is the most effective chemotherapeutic agent available against multiple histological subtypes [68]. The maximum cumulative dose that should be administered should not extend $550 \mathrm{mg} / \mathrm{m}^{2}$ to avoid cumulative cardiotoxicity. Pegylated liposomal doxorubicin has similar efficacy with an improved toxicity profile as compared to doxorubicin in a phase II trial. However response rates were lower than normally expected (ca. 10\%), probably because of the high proportion of GIST in this study population [69].

The second most commonly used drug in soft tissue sarcoma is ifosfamide. Used as monotherapy, ifosfamide results in response rates of $20-25 \%$ in non-pretreated patients [70-72]. Ifosfamide has higher activity in synovial sarcoma and less antitumor activity in leiomyosarcoma [73]. The response rate to ifosfamide is both dose- and schedule-dependent [72]. A randomized phase II study comparing standard-dose ifosfamide $5 \mathrm{~g} / \mathrm{m}^{2}$ over $24 \mathrm{~h}$ versus ifosfamide $3 \mathrm{~g} / \mathrm{m}^{2}$ daily for 3 days reported a response rate of $10 \%$ for the lower-dose treatment and $25 \%$ for the higher dose [71]. Therefore, the most commonly used scheme is $3 \mathrm{~g} / \mathrm{m}^{2}$ ifosfamide administered on days 1, 2, and 3, repeated every 3 weeks. A role for high-dose ifosfamide $\left(14-18 \mathrm{mg} / \mathrm{m}^{2}\right)$ has been suggested in the treatment of metastatic synovial sarcoma [74].

A head-to-head comparison of doxorubicin and ifosfamide in first-line treatment for patients with advanced and/or metastatic soft tissue sarcoma found no differences in PFS, OS, or response rates. However, grade 4 toxicities were more frequent in the ifosfamide arms [75]. This finding supports the use of single-agent doxorubicin as the treatment of choice in metastatic STS, though ifosfamide is a reasonable alternative if patients cannot be treated with an anthracycline initially.

Multi-agent chemotherapy with doxorubicin plus ifosfamide in first-line treatment of metastatic STS results in higher overall 
response rate (26.5\% versus $13.6 \%)$, but without survival advantage over single agent doxorubicin [64]. Therefore, combination therapy may be considered only when a tumor response is felt to be potentially advantageous [5].

Ifosfamide may be used after failure of anthracycline-based chemotherapy in patients who did not progress on it previously $[5,13]$. The median survival of patients exposed to ifosfamide in second-line treatment after doxorubicin failure is in the range of 35-45 weeks with a median time-to-progression of $6-14$ weeks [70, 71]. For patients who have already received standard-dose ifosfamide, high-dose ifosfamide is a reasonable option [76, 77].

Other conventional cytotoxic drugs such as dacarbazine [78, 79], temozolomide [80], pacliatxel [81], docetaxel [82-84], gemcitabine [82, 83], and carboplatin [85] have demonstrated modest antitumor activity in pretreated patients with advanced STS (response rate less than 20\%) but yield disease stabilization. Some of these agents have shown the highest antitumor activity in selected histological subtypes such as taxane in angiosarcomas [81, 86], gemcitabine in leiomyosarcoma and angiosarcoma [87-89], dacarbazine in leiomyosarcoma and solitary fibrous tumor [5], and eribulin in liposarcoma and leiomyosarcoma [90]. Thus a personalized treatment based on a histology-driven approach may improve results and patients outcome.

Eribulin has been shown to improve OS by 2 months (13.5 versus 11.5 months) as compared with standard treatment dacarbazine in heavily pretreated patients with advanced liposarcomas or leimyosarcomas. A total of 452 patients have been enrolled in a randomized open label multicenter phase III trial (Study 309). The study's primary end point of OS was met. Eribulin reduced the risk of death by $23 \% \quad(\mathrm{HR}=0.768 ; 95 \% \quad \mathrm{CI}$ $0.618-0.954 ; P=0.017)$. However, secondary end points (PFS) were not significantly different (median PFS was 2.6 months in both arms). The 2 months improvement seen with eribulin must be weighed against the higher rates of adverse events in the eribulin group; neutropenia (44\% versus 24\%), peripheral sensory neuropathy (20\% versus $4 \%$ ), pyrexia (28\% versus $14 \%$ ), and alopecia (35\% versus $3 \%$ ), with higher rates of grade 3 (63\% versus $53 \%)$, grade 4 (26\% versus $20 \%$ ), and toxic death (4\% versus $1 \%$ ) [91].

Trabectedin is a new agent that acts by binding to the minor groove of the DNA double strand and blocks the cell cycle in late $S$ and $G$ phases. Trabectedin results in a low response rate (8\%) but yields prolonged disease stabilization. Leiomyosarcoma and myxoid liposarcoma appear to be more sensitive to trabectedin. A particularly higher activity was described in myxoid liposarcoma [92, 93]. It is approved for advanced previously treated STS in Europe on the basis a randomized phase II trial [93]. A recent phase III trial comparing trabectedin with dacarbazine in patients with liposarcoma and leiomyosarcoma confirms the results from the prior phase II study. The trial was conducted in 518 patients who previously received an anthracycline-containing regimen followed by at least one additional line of chemotherapy. The primary end point was OS. Secondary outcome measures included PFS, objective response rate, and safety. There was a highly statistically significant difference in PFS (4.2 months with trabectedin versus 1.5 months with dacarbazine $\mathrm{HR}=0.55 ; \quad P<0.0001)$. However, this trial found no improvement in OS (median OS was 12.4 months with trabectedin versus 12.9 months with dacarbazine $(\mathrm{HR}=0.87$; 95\% CI 0.644-1.181; 
$P=0.374)$. The safety profiles were consistent with the well-characterized toxicities of both drugs [94].

Several other multi-agent combinations of active drugs in STS have been investigated. Doxorubicin plus dacarbazine leads to a response rate of $30 \%$ without a benefit in terms of OS [95, 96]. This regimen is a reasonable choice in the first-line treatment of leiomyosarcoma which is less sensitive to ifosfamide [5], or in patients in whom ifosfamide is contraindicated.

The combination of gemcitabine plus docetaxel is widely used in second-line treatment especially in leiomyosarcoma and undifferentiated pleomorphic sarcoma. The most relevant trial in this field has compared gemcitabine with and without docetaxel in patients with advanced STSs. This phase II randomized trial showed the combination to be superior to single-agent gemcitabine in terms of response rate, but also in terms of PFS and OS, but with increased toxicity [83].

The combination of gemcitabine and docetaxel shows no advantage over standard of care doxorubicin as first-line treatment of advanced STS. A recent prospective randomized controlled phase III trial compared this combination with single-agent doxorubicin as first-line treatment in advanced unresectable or metastatic STS. A total of 257 patients were enrolled. The primary end point was PFS rate (PFR) at 24 weeks. In the doxorubicin group $46.1 \%$ of patients were progression-free at 24 weeks versus $46 \%$ in the gemcitabine plus docetaxel group. The HR indicated superiority of doxorubicin ( $\mathrm{HR}=1.28$; 95\% CI 0.98-1.67; $P=0.07)$. Median OS was 71 weeks versus 63 weeks $(\mathrm{HR}=1.07 ; 95 \%$ CI $0.77-1.49)$ for doxorubicin versus gemcitabine plus docetaxel, respectively. Thus, doxorubicin remains the standard first-line treatment for locally advanced/metastatic STS [97].

The combination of dacarbazine and gemcitabine was shown to improve the OS (16.8 versus 8.2 months) and PFS (4.2 versus 2 months) over dacarbazine in 113 patients with previously pretreated STS [98].

\section{TARGETED THERAPIES}

Pazopanib is an oral kinase inhibitor targeting VEGF-R, PDGFR, and c-KIT. It is the first and the only antiangiogenic drug approved for the treatment of refractory non-adipocytic soft tissue sarcoma [99]. After promising results in a phase II trial [100], a large randomized phase III trial (PALETTE) was conducted. The PALETTE trial showed a benefit in terms of PFS averaging 3 months (median 4.6 versus 1.6 months; $P<0.0001)$ for pazopanib given up to progression in refractory non-adipocytic soft tissue sarcoma patients [101]. However, no significant benefit in terms of OS was found; the median OS in patients treated with pazopanib was 12.5 versus 10.7 months in the placebo arm $(P=0.25)$. This was explained by the use of post-trial systemic therapy with other agents in the placebo group. The objective response rate was $6 \%$ for pazopanib versus $0 \%$ for placebo, with $67 \%$ stable diseases in the pazopanib arm versus $38 \%$ in the placebo arm [101]. In the PALETTE trial, adipogenic tumors were excluded on the basis of the lack of activity of pazopanib in this histology subtype in the phase II trial. However, an ongoing trial (ClinicalTrials identifier NCT1506596) will assess pazopanib's activity in adipocytic sarcomas including dedifferentiated, myxoid-round cell, pleomorphic, and mixed type [102], since these genetic subtypes have vascular patterns and may theoretically respond 
to pazopanib [102]. The most common adverse events of pazopanib were fatigue, diarrheas, nausea, weight loss, and hypertension [101]. Retrospective analysis on pooled data from the previously cited phase II and III EORTC trials showed that good performance status, low/ intermediate grade of the primary tumor, and a normal hemoglobin level at baseline were advantageous for long-term outcome. Long-term responders were defined as patients with PFS of at least 6 months (36\%), long-term survivors as patients who survived for at least 18 months (34\%) [103].

There is some evidence of the activity of several molecular targeted agents, including tyrosine kinase inhibitors and mammalian target of rapamycin (mTOR) inhibitors in selected histologies. However, these agents are not approved by regulatory authorities for the treatment of STS and should be preferably used within clinical trials [13].

Cediranib, a potent inhibitor of VEGFR receptors, has shown activity in alveolar soft part sarcoma (ASPS) with a disease control rate at 24 weeks of $84 \%$ in a phase II trial [104].

Sunitinib, an oral angiogenesis inhibitor, achieved promising results in patients with solitary fibrous tumors $(n=10)$ with $70 \%$ objective response and response duration of more than 6 months in five cases [105]. Sunitinib has also shown clinical efficacy in five of nine patients with ASPS treated with sunitinib $37.5 \mathrm{mg}$ daily, continuously [106].

Crizotinib, an orally ATP-competitive inhibitor of the ALK and MET tyrosine kinases, has shown antitumor activity in ALK-rearranged inflammatory myofibroblastic tumor [107].

Regorafenib, a multikinase inhibitor, demonstrated promising activity and an acceptable toxicity profile in a recent randomized placebo-controlled phase II study
(REGOSARC). The trial included 110 patients with metastatic STS. The patients were previously treated with doxorubicine, ifosfamide, trabectedin, or pazopanib (median of prior lines 2, range 1-3). The median PFS of leimyosarcoma patients was 4 months with regorafenib versus 1.9 months with the placebo $\quad(\mathrm{HR}=0.49 ; \quad 95 \% \quad$ CI $\quad 0.27-0.89$; $P=0.017)$ and 4.6 months versus 1.0 month with regorafenib and placebo, respectively $(\mathrm{HR}=0.38 ; 95 \%$ CI $0.20-0.74 ; P=0.002)$ in other types of STS [108].

Ridaforolimus, an mTOR inhibitor, has been tested in a phase II trial conducted in 213 patients with advanced STS. Out of 193 patients with an evaluable response, 28\% showed clinical benefit. These encouraging results led to a phase III trial (SUCCEED) which investigated maintenance therapy with ridaforolimus after chemotherapy in patients with metastatic STS. The PFS was improved with $52 \%$ gain in median PFS (22.4 weeks for ridaforolimus versus 14.7 weeks for placebo; $\mathrm{HR}=0.72 ; P<0.001)$. However, this trial failed to show a benefit in OS.

Sirolimus, another mTOR inhibitor, has resulted in significant clinical activity in patients with malignant perivascular epithelioid cell tumors (PEComa) through a mechanism involving the mTOR1 pathway, pathologically activated by loss of TSC1/TSC2 tumor suppressor complex in PEComa [109].

More recently, olaratumab, a human anti-platelet-derived growth factor alpha $(\mathrm{PDGF} \alpha)$ monoclonal antibody has shown promising results in the treatment of advanced STS. It is the first agent added to doxorubine to achieve an improvement in $\mathrm{OS}(\mathrm{HR}=0.44$; $P=0.0005)$ in a randomized phase II trial [110].

Despite a significant number of phase II trials of targeted therapies, a limited number of agents are tested in phase III trials. 
Determining the optimal trial design and identifying the predictive biomarkers are crucial steps for the development of these drugs.

\section{CONCLUSIONS}

The management of adult STS is complex and should be carried out in a center with expertise in the treatment of sarcomas. A multidisciplinary approach is required for optimal outcome. Clinical guidelines still face some uncertainty given the heterogeneity of the available data. New methods for clinical trials are needed to generate reliable evidence for standard practice.

\section{ACKNOWLEDGMENTS}

No funding or sponsorship was received for publication of this article. All named authors meet the International Committee of Medical Journal Editors (ICMJE) criteria for authorship for this manuscript, take responsibility for the integrity of the work as a whole, and have given final approval for the version to be published.

Disclosures. Samia Arifi, Rhizlan Belbaraka, Rabie Rahhali, and Nabil Ismaili have nothing to disclose.

Compliance with ethical standards. This article is based on previously conducted studies and does not involve any new studies of human or animal subjects performed by any of the authors.

Open Access. This article is distributed under the terms of the Creative Commons Attribution-NonCommercial 4.0 International License (http://creativecommons.org/licenses/ by-nc/4.0/), which permits any noncommercial use, distribution, and reproduction in any medium, provided you give appropriate credit to the original author(s) and the source, provide a link to the Creative Commons license, and indicate if changes were made.

\section{REFERENCES}

1. Fletcher CDM, Bridge JA, Hogendoorn PCW, Lyon MF. World Health Organization classification of tumours of soft tissue and bone. 4th ed. Lyon: IARC Press; 2013.

2. Jemal A, Tiwari RC, Murray T, et al. Cancer statistics. CA Cancer J Clin S. 2004;54:8-29.

3. Siegel RL, Miller KD, Jemal A. Cancer statistics. CA Cancer J Clin. 2015;65(1):5-29.

4. Lawrence W Jr, Donegan WL, Ntarajan N, Mettlin C, Beart R, Winchester D. Adult soft tissue sarcomas. A pattern of care survey of the American College of Surgeons. Ann Surg. 1987;205(4):349-59.

5. The ESMO/European Sarcoma Network Working Group. Soft tissue and visceral sarcomas: ESMO Clinical Practice Guidelines for diagnosis, treatment and follow-up. Ann Oncol. 2014;25 Suppl 3:iii102-iii112.

6. Christie-Large M, James SL, Tiessen L, Davies AM, Grimer RJ. Imaging strategy for detecting lung metastases at presentation in patients with soft tissue sarcomas. Eur J Cancer. 2008;44(13):1841-5.

7. Fong Y, Coit DG, Woodruff JM, Brennan MF. Lymph node metastasis from soft tissue sarcoma in adults. Analysis of data from a prospective database of 1772 sarcoma patients. Ann Surg. 1993;217(1):72.

8. Edge SB, Byrd DR, Compton CC, et al. AJCC (American Joint Committee on Cancer). Cancer staging manual. 7th ed. New York: Springer; 2010, p 291.

9. Zagars GK, Ballo MT, Pisters PW, et al. Prognostic factors for patients with localized soft-tissue sarcoma treated with conservation surgery and radiation therapy: an analysis of 1225 patients. Cancer. 2003;97(10):2530.

10. Gustafson P, Dreinhofer KE, Rydholm A. Soft tissue sarcoma should be treated at a tumor 
center. A comparaison of quality of surgery in 375 patients. Acta Orthop Scand. 1994;65(1):47.

11. Trovik CS, Bauer HC, Alvegrad TA, et al. Surgical margins, local recurrence and metastasis in soft tissue sarcomas: 559 surgically-treated patients from the Scandinavian Sarcoma Group Register. Eur J Cancer. 2000;36(6):710-6.

12. Stoeckle E, Coindre JM, Kantor G, et al. Quality of surgery in soft tissue sarcoma: a single centre experience with the French Sarcoma Group (FSG) surgical system. Cancer therapy. 2005;3:31-40.

13. National Comprehensive Cancer Network. Soft tissue sarcoma (Version 1. 2015). http://www. nccn.org/professionals/physician_gls/pdf/sarcoma. pdf. Accessed 15 May 2015

14. Rosenberg SA, Tepper J, Glastein E, et al. The treatment of soft tissue sarcomas of the extremities: prospective randomized evaluations of (1) limb-sparing surgery plus radiation therapy compared with amputation and (2) the role of adjuvant chemotherapy. Ann Surg. 1982;196(3): 305-15.

15. Rosenberg SA, Tepper J, Glatskin E, et al. Prospective randomized evaluation of adjuvant radiotherapy in adults with soft tissue sarcomas of the extremities. Cancer. 1983;52:424-34.

16. Keus RB, Rutgers EJ, Ho GH, Gortzak E, Albus-lutter CE, Hart AA. Limb-sparing therapy of extremity soft tissue sarcomas: treatment outcome and long term functional results. Eur J Cancer. 1994;30A(10):1459-63.

17. Delaney TF, Harmon DC, Gebhardt MC. Local treatment for primary soft tissue sarcoma of the extremities and chest wall. In: Post TW, editor. Up ToDate. Up ToDate: Waltham, MA; 2015.

18. Maduekwe UN, Hornicek FJ, Springfield DS, et al. Role of sentinel lymph node biopsy in the staging of synovial, epitheloid, and clear cell sarcomas. Ann Surg. 2009;15(5):1356.

19. Andreou D, Boltdt H, Werner M, Hamann C, Pink D, Tunn PU. Sentinel node biopsy in soft tissue sarcoma subtypes with high propensity for regional lymphatic spread-results of a large prospective trial. Ann Oncol. 2013;25(5):1400-5.

20. Baldini EH, Gildgerg J, Jenner C, et al. Long term outcomes after function-sparing surgery without radiotherapy for soft tissue sarcoma of the extremities and trunk. J Clin Oncol. 1999;17(10): 3252.

21. Pisters PW, Pollock RE, Lewis VO, et al. Long-term results of prospective trial of surgery alone with selective use of radiation for patients with T1 extremity and trunk soft tissue sarcomas. Ann Surg. 2007;246(4):675.

22. Cahlon O, Brennan MF, Jia X, Qin LX, Singer S, Atektiar KM. A prospective nomogram for local recurrence risk in extremity soft tissue sarcomas after limb-sparing surgery without adjuvant radiation. Ann Surg. 2012;255(2):343.

23. Yang JC, Chang AE, Baker AR, et al. Randomized prospective study of the benefit of adjuvant radiation therapy in the treatment of soft tissue sarcomas of the extremity. J Clin Oncol. 1998;16(1):197.

24. Pisters PW, Harisson LB, Leung DH, Woodruff JM, Casper ES, Bernnan MF. Long-term results of prospective randomized trial of adjuvant brachytherapy in soft tissue sarcoma. J Clin Oncol. 1996;14(3):859.

25. Pisters PW, Leung DH, Woodruff J, Shi W, Brennan MF. Analysis of prognostic factors in 1041 patients with localized soft tissue sarcomas of the extremities. J Clin Oncol. 1996;14(5):1679-89.

26. Trovik CS, Bauer HC, Alvegård TA, et al. Surgical margins, local recurrence and metastasis in soft tissue sarcomas: 559 surgically-treated patients from the Scandinavian Sarcoma Group Register. Eur J Cancer. 2000;36(6):710-6.

27. Zagars GK, Ballo MT, Pisters PW, Pollock RE, Patel SR, Benjamin RS. Surgical margins and reresection in the management of patients with soft tissue sarcoma using conservative surgery and radiation therapy. Cancer. 2003;97(10):2544-53.

28. Khanfir K, Alzieu L, Terrier P, et al. Does adjuvant radiation therapy increase loco-regional control after optimal resection of soft-tissue sarcoma of the extremities. Eur J Cancer. 2003;39(13):187280.

29. Coindre JM, Terrier P, Bui NB, et al. Prognostic factors in adult patients with locally controlled soft tissue sarcoma. A study of 546 patients from the French Federation of Cancer Centers Sarcoma Group. J Clin Oncol. 1996;14(3):869-77.

30. O'Sullivan B, Davis AM, Turcotte R, et al. Preoperative versus postoperative radiotherapy in soft-tissue sarcoma of the limbs: a randomised trial. Lancet. 2002;359(9325):2235.

31. O'Sullivan B, Davis AM, Turcotte R, et al. Five-year results of a randomized phase III trial of pre-operative versus postoperative radiotherapy in extremity soft tissue sarcoma. J Clin Oncol. 2004;22(14 suppl):9007. 
32. O'Sullivan B, Griffin AM, Dickie CI, et al. Phase 2 study of preoperative image-guided intensity-modulated radiation therapy to reduce wound and combined modality morbidities in lower extremity soft tissue sarcoma. Cancer. 2013;119(10):1878-84.

33. Lindberg RD, Martin RG, Romsdahl MM, Barkley HT Jr. Conservative surgery and postoperative radiotherapy in 300 adults with soft-tissue sarcomas. Cancer. 1981;47(10):2391.

34. Sarcoma Meta-analysis Collaboration. Adjuvant chemotherapy for localised resectable soft-tissue sarcoma of adults: meta-analysis of individual data. Lancet. 1997;350(9092):1647-54.

35. Pervaiz N, Colterjohn N, Farrokhyar F, Tozer R, Figueredo A, Ghert M. A systematic meta-analysis of randomized controlled trials of adjuvant chemotherapy for localized resectable soft-tissue sarcoma. Cancer. 2008;113(3):573-81.

36. Woll PJ, Reichardt P, Le Cesne A, et al. EORTC Soft Tissue and Bone Sarcoma Group and the NCIC Clinical Trials Group. Adjuvant chemotherapy with doxorubicin, ifosfamide, and lenograstim for resected soft-tissue sarcoma (EORTC 62931): a multicentre randomised controlled trial. Lancet Oncol. 2012;13(10):1045-54.

37. Le Cesne A, Ouali $M$, Leahy $M G$, et al. Doxorubicin-based adjuvant chemotherapy in soft tissue sarcoma: pooled analysis of two STBSG-EORTC phase III clinical trials. Ann Oncol. 2014;25(12):2425-32.

38. Jones RL, Fisher C, Al-Muderis O, Judson IR. Differential sensitivity of liposarcoma subtypes to chemotherapy. Eur J Cancer. 2005;41(18):2853.

39. Rosen G, Forscher C, Lowenbraun S, et al. Synovial sarcoma. Uniform response of metastases to high dose ifosfamide. Cancer. 1994;73(10):2506.

40. Ferrari A, Gronchi A, Casanova M, et al. Synovial sarcoma: a retrospective analysis of 271 patients of all ages treated at single institution. Cancer. 2004;101(3):627.

41. Eilber FC, Eilber FR, Eckardt J, et al. The impact of chemotherapy on the survival of patients with high grade primary extremity liposarcoma. Ann Surg. 2004;240(4):686.

42. Angele MK, Albertsmeier M, Prix NJ, et al. Effectiveness of regional hyperthermia with chemotherapy for high-risk retroperitoneal and abdominal soft-tissue sarcoma after complete surgical resection: a subgroup analysis of a randomized phase-III multicenter study. Ann Surg. 2014;260(5):749-54.
43. Issel RD, Lindner LH, Verweij J, et al. Neo-adjuvant chemotherapy alone or with regional hyperthermia for localized high risk soft tissue sarcoma: a randomised phase 3 multicentre study. Lancet Oncol. 2010;11(6):561-70.

44. Gutman M, Inbar M, Lev-Shulsh D, et al. High dose tumor necrosis factor-alpha and melphalan administered via isolated limb perfusion for advanced limb soft tissue sarcoma result in a $>90 \%$ response and limb preservation. Cancer. 1997;79(6):1129.

45. Eggermont AM, Schraffordt Koops H, Liénard D, et al. Isolated limb perfusion with high-dose tumor necrosis factor-alpha in combination with interferon-gamma and melphalan for nonresectabe extremity soft tissue sarcomas: a multicenter trial. J Clin Oncol. 1996;14(10):2653.

46. Noorda EM, Vrouenraets BC, Nieweg OE, van Coevorden F, van Slooten GW, Kroon BB. Isolated limb perfusion with tumor necrosis factor-alpha and melphalan for patients with unresctable soft tissue sarcoma of the extremities. Cancer. 2003;98(7):1483.

47. Grunhagen DJ, de Wilt JH, Graveland WJ, Verhoef C, van Geel AN, Eggermont AM. Outcome and prognostic factor analysis of 217 consecutive isolated limb perfusions with tumor necrosis factor-alpha and melphalan for limb-threatening soft tissue sarcoma. Cancer. 2006;106(8):1776.

48. Eggermont AM, Schraffordt Koops H, Klausner $\mathrm{JM}$, et al. Isolated limb perfusion with tumor necrosis factor and melphalan for limb salvage in 186 patients with locally advanced soft tissue extremity sarcomas. The cumulative multicenter European experience. Ann Surg. 1996;224(6):756.

49. Issels RD, Abdel-Rahman S, Wendtner $\mathrm{C}$, et al. Neoadjuvant combined with regional hypertermia for locally advanced primary or recurrent high risk adult soft tissue sarcomas of adults: long term results of phase II study. Eur J Cancer. 2001;37(13):1599.

50. Pister PW, Patel SR, Prieto VG, et al. Phase I trial of preoperative doxorubicin based concurrent chemoradiation and surgical resection for localized extremity and body wall soft tissue sarcomas. J Clin Oncol. 2004;22(16):3375.

51. Toma S, Canavese G, Grimaldi A, et al. Concomitant chemo-radiotherapy in the treatment of locally advanced and/or metastatic soft tissue sarcomas: experience of the National Cancer Institute of Genoa. Oncol Rep. 2003;10(3): 641. 
52. Cormier JN, Patel SR, Herzog CE, et al. Concurrent ifisfamide-based chemotherapy and irradiation. Analysis of treatment related toxicity in 43 patients with sarcoma. Cancer. 2001;92(6):1550.

53. Kraybill WG, Harris J, Spiro IJ, et al. Phase II study of neoadjuvant chemotherapy and radiation therapy in the management of high risk, high grade, soft tissue sarcomas of the extremities and body wall. J Clin Oncol. 2006;24(4):619.

54. Gortzak E, Azzarelli A, Buesa J, et al. A randomized phase II study on neo-adjuvant chemotherapy for high-risk adult soft tissue sarcoma. Eur J Cancer. 2001;37:1096-103.

55. Antman K, Crowley J, Blacerzak SP, et al. An intergroup phase III randomized study of doxorubicin and dacarbazine with or without ifosfamide and mesna in advanced soft tissue sarcomas. J Clin Oncol. 1993;11(7):1276.

56. Elias A, Ryan L, Sulkes A, Collins J, Aisner J, Antman KH. Response to mesna, doxorubicin, ifosfamide, and dacarbazine in 108 patients with metastatic or unresectable sarcoma and no prior chemotherapy. J Clin Oncol. 1989;7(9):1208.

57. Driscoll DL. Treatment and local control of primary extremity soft tissue sarcomas. J Surg Oncol. 1999;71(3):155.

58. Zagars GK, Ballo MT, Piester PW, Pollock RE, Patel SR, Benjamin RS. Prognostic factors for diseases-specific survival after first relapse of soft tissue sarcomas: analysis of 402 patients with disease relapse after initial conservative surgery and radiotherapy. Int J Radiat Oncol Biol Phys. 2003;57(3):739.

59. Levay J, O'Sullivan B, Catton C, et al. Outcome and prognostic factors in soft tissue sacoma in the adult. Int J Radiat Oncol Biol Phys. 1993;27(5): 1091.

60. Indelicato DJ, Meadows K, Gibbs CP Jr, Morris CG, Scarborough MT, Zlotecki RA. Effectiveness and morbidity associated with reirradiation in conservative salvage management of recurrent soft-tissue sarcoma. Int J Radiat Oncol Biol Phys. 2009;73(1):267.

61. Pearlstone DB, Janjan NA, Feig BW, et al. Re-resection with brachytherapy for locally recurrent soft tissue sarcoma arising in a previously radiated field. Cancer J Sci Am. 1999;5(1):26.

62. Lans TE, Grünhagen DJ, de Wilt JH, Van Geel AN, Eggermont AM. Isolated limb perfusions with tumor necrosis factor and melphalan for locally recurrent soft tissue sarcoma in previously irradiated limbs. Ann Surg Oncol. 2005;12(5):406.

63. Italiano A, Mathoulin-Pelissier S, Le Cesne A, et al. Trends in survival for patients with metastatic soft-tissue sarcoma. Cancer. 2011;117:1049-54.

64. Judson I, Verweij J, Gelderblom $H$, et al. Doxorubicin alone versus intensified doxorubicin plus ifosfamide for first-line treatment of advanced or metastatisoft-tissue sarcoma: a randomised controlled phase 3 trial. Lancet Oncol. 2014;15: 415-23.

65. Blay JY, Van Glabbeke $M$, Verweij J, et al. Advanced soft-tissue sarcoma: a disease that is potentially curable for a subset of patients treated with chemotherapy. Eur J Cancer. 2003;39:64-9.

66. Blackmon SH, Shah N, Roth JA, et al. Resection of pulmonary and extrapulmonary sarcomatous metastases is associated with long-term survival. Ann Thorac Surg. 2009;88(3):877-84.

67. Billingsley KG, Burt ME, Jara E, et al. Pulmonary metatases from soft tissue sarcoma: analysis of patterns of disease and postmetastases survival. Ann Surg. 1999;229(5):602.

68. Bramwell VH, Anderson D, Charette ML. Doxorubicin-based chemotherapy for the palliative treatment of adult patients with locally advanced or metastatic soft-tissue sarcoma: a meta-analysis and clinical practice guideline. Sarcoma. 2000;4:103-12.

69. Judson I, Radford JA, Harris M, et al. Randomised phase II trial of pegylated liposomal doxorubicin (DOXIL/CAELYX) versus doxorubicin in the treatment of advanced or metastatic soft tissue sarcoma: a study by the EORTC Soft Tissue and Bone Sarcoma Group. Eur J Cancer. 2001;37: 870-7.

70. Tascilar M, Loos WJ, Seynaeve C, Verweij J, Sleijfer $\mathrm{S}$. The pharmacologic basis of ifosfamide use in adult patients with advanced soft tissue sarcomas. Oncologist. 2007;12:1351-60.

71. Van Oosterom AT, Mouridsen HT, Nielsen OS, et al. Results of randomised studies of the EORTC Soft Tissue and Bone Sarcoma Group (STBSG) with two different ifosfamide regimens in first- and second-line chemotherapy in advanced soft tissue sarcoma patients. Eur J Cancer. 2002;38:2397-406.

72. Patel S, Vadhan-Raj S, Papadopolous N, et al. High-dose ifosfamide in bone and soft tissue sarcomas: results of phase II and pilot studies-dose response and schedule dependence. J Clin Oncol. 1997;15:2378-84. 
73. Sleijfer $S$, Ouali $M$, van Glabbeke $M$, et al. Prognostic and predictive factors for outcome to first-line ifosfamide-containing chemotherapy for adult patients with advanced soft tissue sarcomas: an exploratory, retrospective analysis on large series from the European Organization for Research. Eur J Cancer. 2010;46(1):72-83.

74. Rosen G, Forscher C, Lowenbraun S, et al. Synovial sarcoma, Uniform response of metastases to high dose ifosfamide. Cancer. 1994;73(10):2506-11.

75. Lorigan PL, Verweij J, Papai Z, et al. Phase III trial of two investigational schedules of ifosfamide compared with standard-dose doxorubicin in advanced or metastatic soft tissue sarcoma: a European Organisation for Research and Treatment of Cancer Soft Tissue and Bone Sarcoma Group Study. J Clin Oncol. 2007;25(21): 3144-50.

76. Le Cesne A, Antoine E, Spielmann $M$, et al. High-dose ifosfamide: circumvention of resistance to standard-dose ifosfamide in advanced soft tissue sarcomas. J Clin Oncol. 1995;13:1600-8.

77. Martin-Liberal J, Alam S, Constantinidou A, et al. Clinical activity and tolerability of a 14-day infusional Ifosfamide schedule in soft-tissue sarcoma. Sarcoma. 2013;868-973.

78. Gottlieb JA, Benjamin RS, Baker LH, et al. Role of DTIC (NSC-45388) in the chemotherapy of sarcomas. Cancer Treat Rep. 1976;60(2):199.

79. Zucali PA, Bertuzzi A, Parra HJ, Campagnoli E, Qualiuolo V, Santoro A. The "old drug" dacarbazine as second/third line chemotherapy in advanced soft tissue sarcomas. Invest New Drugs. 2008;26(2):175.

80. Garcia del Muro X, Lopez-Pousa A, Martin J, et al. A phase II trial of temozolomide as a 6-week, continuous, oral schedule in patients with advanced soft tissue sarcoma: a study by the Spanish Group for Research on Sarcomas. Cancer. 2005;104(8):1706.

81. Penel N, Bui BN, Bay JO, et al. Phase II trial of weekly paclitaxel for unresectable angiosarcoma: the ANGIOTAX Study. J Clin Oncol. 2008;26:5269-74.

82. Pautier P, Floquet A, Penel N, et al. Randomized multicenter and stratified phase II of gemcitabine alone versus gemcitabine and docetaxel in patients with metastatic or relapsed leiomyosarcomas: a Federation Nationale des Centres de Lutte Contre le Cancer French Sarcoma Group Study (TAXOGEM). Oncologist. 2012;17(9):1213-20.
83. Maki RG, Wathen JK, Patel SR, et al. Randomized phase II study of gemcitabine and docetaxel compared with gemcitabine alone in patients with metastatic soft tissue sarcomas: results of sarcoma alliance for research through collaboration study 002. J Clin Oncol. 2007;25(19):2755.

84. Bramwell V, Blackstein $\mathrm{M}$, Belanger $\mathrm{K}$, et al. A phase II study of docetaxel in chemotherapy-naïve patients with recurrent or metastatic adult soft tissue sarcoma. Sarcoma. 1998;2:29-33.

85. Goldstein D, Cheuvart B, Trump DL, et al. Phase II trial of carboplatin in soft tissue sarcoma. Am J Clin Oncol. 1990;13(5):420.

86. Schlemmer M, Reichardt P, Verweij J, et al. Paclitaxel in patients with advanced, angiosarcomas of soft tissue: a retrospective study of the EORTC soft tissue and bone sarcoma group. Eur J Cancer. 2008;44:2433-6.

87. Hartmann JT, Oechsle K, Huober J, et al. An open label, non-comparative phase II study of gemcitabine as salvage treatment for patients with pretreated adult type soft tissue sarcoma. Invest New Drugs. 2006;24:249-53.

88. Von Burton G, Rankin C, Zalupski MM, et al. Phase II trial of gemcitabine as first line chemotherapy in patients with metastatic or unresectable soft tissue sarcoma. Am J Clin Oncol. 2006;29:59-61.

89. Stacchiotti S, Palassini E, Sanfilippo R, et al. Gemcitabine in advanced angiosarcoma: a retrospective case series analysis from the Italian Rare Cancer Network. Ann Oncol. 2012;23:501-8.

90. Schoffski $\mathrm{P}$, et al. Activity of eribulin mesylate in patients with soft-tissue sarcoma: a phase 2 study in four independent histological subtypes. Lancet Oncol. 2011;12:1045-52.

91. Schöffski P, Maki RG, Italiano $A$, et al. Randomized, open-label, multicenter, phase III study of eribulin versus dacarbazine in patients with leiomyosarcoma and adipocytic sarcoma. J Clin Oncol. 2015;33(18 suppl):LBA10502.

92. Le Cesne A, et al. A retrospective pooled analysis of trabectedin safety in 1132 patients with solid tumors treated in phase II clinical trials. Investig New Drugs. 2012;30:1193-202.

93. Demetri GD, Chawla SP, Von Mehren M, et al. Efficacy and safety of trabectedin in patients with advanced or metastatic liposarcoma or leiomyosarcoma after failure of prior anthracyclines and ifosfamide: results of a randomized phase II study of two different schedules. J Clin Oncol. 2009;27:4188-96. 
94. Demetri GD, Mehren VM, Jones RL, et al. A randomized phase III study of trabectidin or dacarbazin for the treatment of patients with advanced liposarcoma or leiomyosarcoma. J Clin Oncol. 2015;33(suppl):10503.

95. Antman K, Crowley J, Balcerzak SP. Randomized comparison of doxorubicin and dacarbazine with or without ifosfamide and mesna in advanced soft tissue sarcomas. J Clin Oncol. 1993;11(7):1269-75.

96. Borden EC, Amato DA, Rosenbaum C, et al. Randomized comparison of three adriamycin regimens for metastatic soft tissue sarcomas. J Clin Oncol. 1987;5:840-50.

97. Seddon BM, Whelan J, Strauss SJ, et al. GeDDis: a prospective randomized controlled phase III trial of gemcitabine and docetaxel compared with doxorubicin as first-line treatment in previously untreated advanced or metastatic soft tissue sarcomas (EudraCT 2009-014907-29). J Clin Oncol. 2015;33(suppl):10500.

98. García-Del-Muro X, López-Pousa A, Maurel J, et al. Randomized phase II study comparing gemcitabine plus dacarbazine versus dacarbazine alone in patients with previously treated soft tissue sarcoma: a Spanish Group for Research on Sarcomas study. J Clin Oncol. 2011;29:2528-33.

99. Kasper B, Sleijfer S, Litière $S$, et al. Long-term responders and survivors on pazopanib for advanced soft tissue sarcomas: subanalysis of two European Organization for Research and Treatment of Cancer (EORTC) clinical trials 62043 and 62072. Ann Oncol. 2014;25:719-24.

100. Sleijfer S, Ray-Coquard I, Papai Z, et al. Pazopanib, a multikinase angiogenesis inhibitor, in patients with relapsed or refractory advanced soft tissue sarcoma: a phase II study from the European Organisation for Research and Treatment of Cancer-Soft Tissue and Bone Sarcoma Group (EORTC) Study 620. J Clin Oncol. 2009;27.

101. van der Graaf WT, Blay JY, Chawla SP, et al. EORTC Soft Tissue and Bone Sarcoma Group; PALETTE study group. Pazopanib for metastatic soft-tissue sarcoma (PALETTE): a randomised, double-blind, placebocontrolled phase 3 trial. Lancet. 2012;379:1879-86.
102. Endo M, Nielsen TO. Pazopanib for metastatic soft-tissue sarcoma. Lancet. 2012;380(9844):801 (author reply 801).

103. Kasper B, Sleijfer S, Litière $S$, et al. Long-term responders and survivors on pazopanib for advanced soft tissue sarcomas: subanalysis of two European Organisation for Research and Treatment of Cancer (EORTC) clinical trials 62043 and 62072. Ann Oncol. 2014;25:719-24.

104. Kummar S, Allen D, Monks A, et al. Cediranib for metastatic alveolar soft part sarcoma. J Clin Oncol. 2013;31(18):2296-302.

105. Stacchiotti S, Negri T, Palassini E, et al. Sunitinib malate and figitumumab in solitary fibrous tumor: patterns and molecular bases of tumor response. Mol Cancer Ther. 2010;9(5):1286-97.

106. Stacchiotti S, Tamborini E, Marrari A, et al. Response to sunitinib malate in advanced alveolar soft part sarcoma. Clin Cancer Res. 2009;15:1096-104.

107. Butrynski JE, D'Adamo DR, Hornick JL, et al. Crizotinib in ALK-rearranged inflammatory myofibroblastic tumor. $\mathrm{N}$ Engl J Med. 2010;363(18):1727-33.

108. Mir O, Brodowicz T, Wallet J, et al. Activity of regorafenib in leimyosarcomas and other types of soft tissue sarcomas: results of double- blind, randomized placebo controlled phase II trial. J Clin Oncol. 2015;33(suppl):10504.

109. Wagner AJ, Malinowska-Kolodziej I, Morgan JA, et al. Clinical activity of mTOR inhibition with sirolimus in malignant perivascular epithelioid cell tumors: targeting the pathogenic activation of mTORC1 in tumors. J Clin Oncol. 2010;28(5): 835-40.

110. Tap WD, Jones RL, Chmielowski B, et al. A randomized phase Ib/II study evaluating safety and efficacy of olaratumab (IMC-303) a human anti.platelet derived growth factor a (PDGFRa) monoclonal antibody with or without doxorubicin in advanced STS. J Clin Oncol. 2015;33(suppl):10501. 